\title{
Analysis methods for Milky Way dark matter halo detection
}

\author{
Aaron Sander*, Larry Wai ${ }^{\dagger}$, Brian Winer*, Richard Hughes* and Igor V. \\ Moskalenko** \\ *Department of Physics, The Ohio State University, Columbus, OH 43210 \\ ${ }^{\dagger}$ Kavli Institute for Particle Astrophysics and Cosmology, Menlo Park, California 94025 \\ ${ }^{* *}$ HEPL and KIPAC, Stanford Univerisity, Stanford, CA 94305
}

\begin{abstract}
We present methods for the analysis of dark matter annihilation in the smooth halo of the Milky Way galaxy. We model the diffuse gamma-ray background using GALPROP, and model the halo using an NFW profile and the gamma-ray spectrum for WIMP pair annihilation. We plan to combine these models with the point source catalog and a simple model for the extragalactic gamma ray background. Using the downhill simplex method to converge on the maximum likelihood value, we can vary key parameters in these models and fit them to the gamma-ray data. Through the use of the Markov Chain Monte Carlo (MCMC) method we can then map out the likelihood as a function of the model parameters to estimate the correlated errors on these parameters.
\end{abstract}

\section{INTRODUCTION}

Dark matter evidence comes primarily from rotation curve data and cluster dynamics. Potential dark matter candidates include axions, black holes, MACHOS, WIMPs, and others[1]. Dark Matter currently makes up 20\% of the energy density of the universe. One of the currently favored models is one in which the WIMP is the lightest supersymmetric particle, called the neutralino. This particle is theorized to be a Majorana particle and this property would allow for indirect detection by GLAST via pair annihilation to q-qbar pairs (continuum), Z gamma, or gamma gamma (lines).

Popular models for the density profile include isothermal [2], NFW [3], Kravtsov [4], and Moore [5]. One parameterization of the density that allows all three to be included is

$$
\rho(r)=\frac{\rho_{0}}{(r / R)^{\gamma}\left[1+(r / R)^{\alpha}\right]^{(\beta-\gamma) / \alpha}}
$$

where $R$ is the characteristic scale radius and $\rho_{0}$ is the characteristic density of the halo. Using this parameterization we recover the NFW profile if we set $\alpha=1, \beta=3, \gamma=1$, and $R=20 \mathrm{kpc}$ [3].

\section{METHODS}

We generated two skymap models to represent the distribution of flux in the sky. The model for galactic diffuse gamma ray emission was generated with Galprop [6] simulation. The dark matter model was generated using a NFW density profile with gamma ray spectra generated from PYTHIA with the WIMPs annihilating to a b-bbar pair. The total flux fixed at $30 \%$ of diffuse total flux above one $\mathrm{GeV}$.

We generated a sample of gamma ray events from the galactic diffuse background skymap and a dark matter halo skymap centered on the galactic center. These events were generated using gtobssim, a fast data simulation program for GLAST data, are shown in the counts map in figure 1. Then, using the same skymaps we fit to the simulated counts from gtobssim. The results of the fit are shown in figure 1. This fit allows us to check that the relative normalizations of the two skymaps and obtain a maximum likelihood value.

In the future, we plan to use the downhill simplex algorithm [7] to vary the model parameters: cross section and neutralino mass for the dark matter halo model; proton injection spectral index and electron injection spectral index for the diffuse model. Using these newly generated models, we will then fit them to our generated counts and obtain a $\log$ likelihood value. This process of varying the model parameters would then be repeated until we have mapped out the likelihood profile, $L_{1}$, distribution as a function of our model parameters. 

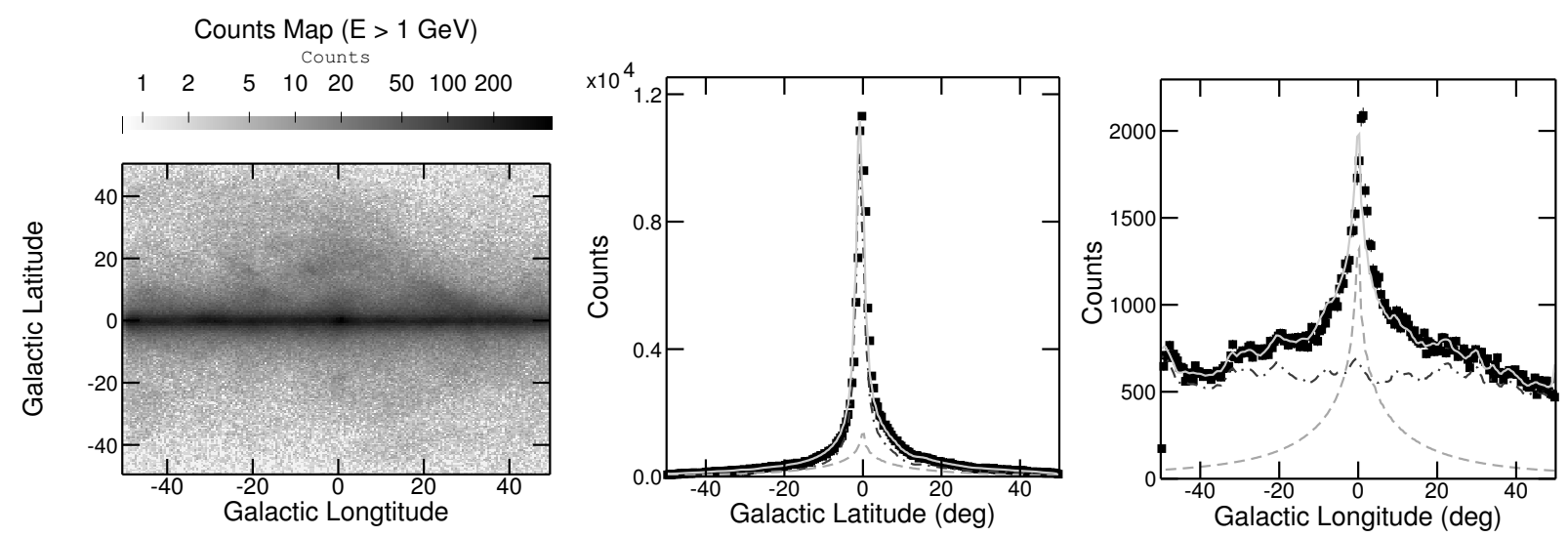

FIGURE 1. On the left panel, the plot shows the gtobssim counts from dark matter annihilations and galactic diffuse gamma ray emission. On the center and right panels, the plots contain the fit to gtobssim simulated data (black points) for diffuse background (dash-dotted line), dark matter halo (dashed line) and the sum (solid line).

We can then obtain the errors on the parameters by noting that on $\frac{1}{2}$ unit in log likelihood will give the one sigma errors for that parameter. Also, we can use only the diffuse model to fit the distribution of dark matter plus diffuse, obtaining a second likelihood profile, $L_{0}$, and use the likelihood ratio test to create a test statistic assumed to be distributed as chi-squared [8]:

$$
T_{s}=-2\left(\ln L_{0}-\ln L_{1}\right)
$$

With this test statistic we can set limits on detection of a dark matter signal within our sample.

Presently, we have only used two skymaps: the diffuse and dark matter skymaps. We plan to expand this analysis to include the EGRET catalog sources and the extragalactic diffuse. We plan to start with four model parameters: cross section for the WIMP annihilation, the WIMP mass, the proton injection spectral index for the diffuse model, and the electron injection spectral index for the diffuse model. Also, we will look at various dark matter profiles by choosing specific sets of $\alpha, \beta, \gamma$, and $R$.

IVM is supported in part by a NASA APRA grant.

\section{REFERENCES}

1. G. Bertone, D. Hooper, and J. Silk, Phys.Rept. 405, 279-390 (2005).

2. L. Bergström, P. Ullio, and J. H. Buckley, Astropart. Phys. 9, 137-162 (1998).

3. J. F. Navarro, C. S. Frenk, and S. D. M. White, Astrophys. J. 462, 563 (1996), a stro-ph/9508025.

4. A. V. Kravtsov, A. A. Klypin, J. S. Bullock, and J. R. Primack, Astrophys. J. 502, 48 (1998).

5. B. Moore, T. Quinn, F. Governato, J. Stadel, and G. Lake, Mon. Not. Roy. Astron. Soc. 310, 1147-1152 (1999), astro-ph/9903164.

6. A. W. Strong, and I. V. Moskalenko, Astrophys. J. 509, 212-228 (1998), a stro-ph/980 7150.

7. W. H. Press, B. P. Flannery, S. A. Teukolsky, and W. T. Vetterling, Numerical Recipes in C, Cambridge University Press, New York, NY, 1992, p. 460.

8. J. R. Mattox, D. L. Bertsch, J. Chiang, B. L. Dingus, S. W. Digel, J. A. Esposito, J. M. Fierro, R. C. Hartman, S. D. Hunter, G. Kanbach, D. A. Kniffen, Y. C. Lin, D. J. Macomb, H. A. Mayer-Hasselwander, P. F. Michelson, C. von Montigny, R. Mukherjee, P. L. Nolan, P. V. Ramanamurthy, E. Schneid, P. Sreekumar, D. J. Thompson, and T. D. Willis, Astrophys. J. 461, 396 (1996). 